\title{
Modeling and Calculation of Cyclic Fatigue Life of Surface Plastic Strain Hardened Parts of Mining Machines Based on the Mechanics of Technological Inheritance
}

\author{
Valeriy Yu. Blumenstein \\ T.F. Gorbachev Kuzbass State Technical University \\ Kemerovo, Russian Federation \\ blumenstein@rambler.ru
}

\author{
Oleg A. Ostanin \\ T.F. Gorbachev Kuzbass State Technical University \\ Kemerovo, Russian Federation \\ ooa.tma@kuzstu.ru
}

\begin{abstract}
The study reviews the developed model of deformations accumulation and depletion of the metal plasticity reserve at the cyclic fatigue life stage. Such model is based on the ideas relating to the index of stress state scheme and consistent in terms of physics. It is shown that the type and numerical value of deformation parameters vary widely depending on the degree of hardening and the tensor of inheritable residual stresses. The experimental study was carried out with the calculations to follow and it is shown that a set of methods developed in a context of a loading history is highly reliable.
\end{abstract}

Keywords - hardening, surface plastic strain, cyclic fatigue life, mechanics of technological inheritance, degree of shear strain, degree of plasticity reserve depletion

\section{INTRODUCTION}

It is known that in most cases the cause of failure of machine parts are fatigue fractures. A significant number of parts of ship, tractor and automobile engines, torsion shafts, axles of railway cars and electric locomotives, crank pins, reducers of agricultural, road and mining machines, etc. work in the conditions of variable loads and stresses, that is why ensuring the specified life of these parts remains to be a very urgent problem [1]. In the machine-building industry of the region, on the one hand, the efforts are made to increase the service life of mining machines and, on the other hand, to create conditions for maintenance. Crisis developments resulted in the need to create new and develop existing service centers, their task being to restore and repair, and also to manufacture spare parts. This is especially important for mining machines used for underground coal mining. Here, the share of imported equipment is as follows: coal miners $87 \%$, powered supports $53 \%$, roadheaders $59 \%$, face conveyors $80 \%$.

The choice of manufacturing technology is determined by the laws of fatigue failure of machine parts. Numerous studies have shown that fatigue failure is observed mainly in the surface layers of machine parts [1-3]. Many researchers acknowledge that in the process of fatigue loading, the stress state is not homogeneous; the components of the acting stresses change due to the relaxation of the residual stresses and the gradual accumulation of damage, which leads to a change in the intensity of this process [4]. The prehistory of fatigue loading affects the ductility and fatigue life of the material during subsequent loading along different trajectories [5]. It is noted that if the parameters of nonlinearity of fatigue loading are different, then different load histories will lead to different results [6]. Various criteria of cyclic destruction are used, including, the one based on the idea of the damaging role of microstresses on the way of elastic and plastic deformation; this criterion evaluates the degree of damage to parts taking into account the loading history [7]. Based on the mechanics of destruction and the energy condition, a FEM-model of fatigue damage was developed; the model takes into account the plastic hardening and healing of defects during the evolution of fatigue [8]. The need for modern light metals required an accurate description of their cyclic inelastic behavior under various loading histories. It is stated that cyclic stress relaxation and accumulated deformation are the key investigated phenomena [9]. In the study [10], nonlinear models of fatigue failure of metals under various loading conditions are considered. In the study [11], the growth of a fatigue crack is considered as a process of successive increments of defects in view of the history of stress; therefore, the initiation of the fatigue crack and its subsequent growth is modeled as one repetitive process. A model of the fatigue crack growth (UniGrow model) is obtained, based on the analysis of cyclic elastic-plastic stresses-deformations near the crack tip. The study in which the joint effect of hardening and residual stresses on the formation of working stresses and the endurance limit is evaluated is of interest [12]. Deformation of the surface layer during ball rolling creates in it such zones (deformation zones) which, when external cyclic loads are applied to the part, begin to deform first, and the process of accumulation of damages in them takes place more quickly. It is assumed that the zone around a particularly stressed point is characterized by a certain level of damage achieved during ball rolling, then the accumulation of fatigue damage begins already at this level. When a certain critical value of damage is reached, a fatigue crack develops. An analytical solution is proposed for estimating deformations in the process of cyclic loading under conditions of an inhomogeneous stress state [13]. The results obtained are particular due to the record of residual stresses noted during cyclic elastoplastic deformation.

Numerous studies and practice have shown that longevity in a substantial measure is determined by the quality of the 
surface layer of machine parts. Surface plastic strain (SPS) belongs to the number of effective methods that ensure minimum roughness with an optimal profile, hardening and compressive residual stresses in the surface layer of machine parts [14]. As a result of SPS treatment, the fatigue resistance of smooth carbon steel samples rises from 15 to $48 \%$, that of alloyed steels from 15 to $81 \%$; an increase was noted in the endurance limit after SPS treatment of aluminum alloys from 23 to $33 \%$, of magnesium alloys from 12 to $24 \%$, and of titanium alloys from 10 to $14 \%$. The effect of hardening samples with stress concentrators is particularly of 2.5-3.0 or more times.

By now, the fatigue properties and regularities of the behavior of various materials in the processes of fatigue loading with various forms of cycles are described in the categories of mechanical properties of materials, structural and technological parameters of machine parts after treatment with various methods, including SPS. The results are obtained showing the effect of the entire spectrum of quality parameters of the surface layer on the characteristics of fatigue life, namely, roughness, microstructure, hardening and residual stresses. It has been established that the SPS treatment not only slows down the growth of fatigue cracks, but under certain conditions leads to a complete stoppage of their spread [15-20].

The common techniques of calculation for fatigue presuppose knowledge of the cycle stresses, the safety factors of the strength, the mechanical properties of the material, the unit parameters of the quality of the surface layer, etc. However, after a number of machining operations involving the SPS processing, the real metal is hardened, there is a complex spreading of properties along the depth of the surface layer, taking into account the technological inheretance. In addition, with identical quality parameters of the surface layer, the history of its loading can vary significantly for different machine parts, which also leads to different parameters of fatigue life. It is also important that in the process of fatigue loading, relaxation processes occur that change both the properties of the material and the loading scheme.

The mechanics of technological inheritance (TI) is developed, which is based on the physical concepts of continuous accumulation of deformation and depletion of the plasticity reserve of the surface layer metal at successive stages of the product life cycle [21-23].

\section{THEORETICAL STUDIES}

The life cycle of the part, including the cutting stages, surface plastic strain and subsequent fatigue loading, was considered. The initial and boundary conditions for solving the problems of the TI mechanics at the stages of cutting and SPS were formulated by carrying out experimental studies in the hereditary setting. In solving the problems of the strain mechanics, the analysis of the stress-strain state (SSS) of the metal along the current lines in the deformation centers was performed. An estimate of the accumulated degree of shear deformation $\Lambda$ and the degree of depletion of the plasticity reserve $\Psi$ was carried out according to the procedure described in [21].

Fatigue loading was represented by two stages: the stage of cyclic fatigue life (CFL) and the stage of cyclic fracture strength (CFS). To describe the continued accumulation of deformations and the depletion of the plasticity reserve at the stage of cyclic fatigue life from the level reached after machining, the deformation mechanics apparatus was used. For the purpose of performing the calculations, an index of the stress state scheme, which is uniform in structure and physical meaning, is proposed for various stages and phases of loading - machining by cutting and surface plastic deformation and fatigue loading.

At the stage of cyclic fatigue life under conditions of symmetrical bending with rotation, the sample is under the action of a shear force. The deformation in which only the component of the stress tensor $\sigma \mathrm{x}$ acting along the principal axis differs from zero, is nothing but a deformation of simple stretching or compression. Therefore, the fatigue (cyclic) stresses in the tensor form were represented as

$$
\left[\mathrm{T} \sigma_{\text {fat }}\right]=\left[\begin{array}{ccc}
\sigma_{x} & 0 & 0 \\
0 & 0 & 0 \\
0 & 0 & 0
\end{array}\right] .
$$

The residual stress tensor was calculated in accordance with the unloading theorem, according to which

$\left[\mathrm{T} \sigma_{\text {res }}\right]=\left[\mathrm{T} \sigma_{\text {def }}\right]-\left[\mathrm{T} \sigma_{\text {elast }}\right]+\left[\mathrm{T} \sigma_{\text {det }}\right]+\left[\mathrm{T} \sigma_{t^{\mathrm{o}}}\right]$,

where $\left[\mathrm{T} \sigma_{\text {def }}\right]$ - tensor of stresses arising in real elastoplastic body when the load is applied; [T $\sigma_{\text {elast }}$ ] tensor of stresses arising which would arise in ideally elastoplastic body at identical loading; [T $\left.\sigma_{\text {det }}\right]-$ stress tensor of elastic unloading at detachment of parts; $\left[\mathrm{T} \sigma_{t^{\circ}}\right]_{-}$ tensor of elastic thermal strains of unloading.

The tensor of hereditary residual stresses for the conditions of the task of a plane deformed state was presented as

$$
\left[\mathrm{T} \sigma_{\mathrm{res}}\right]=\left[\begin{array}{ccc}
\sigma_{x} & \tau_{x y} & 0 \\
\tau_{x y} & \sigma_{y} & 0 \\
0 & 0 & \sigma_{z}
\end{array}\right],
$$

where $\sigma_{x}, \sigma_{y}, \sigma_{z}, \tau_{x y}$-normal and tangential components of residual stresses, respectively.

The resultant stress state was determined by superposition of residual stresses and stresses from loading by external forces. With the directions of the principal stress vectors coinciding for both components and the symmetrical cycle of stresses from the external load, the surface layer of the part operates under conditions of the cycle asymmetry which is the greater with the greater the residual stresses. The average stress of the cycle is then equal to the residual stress, and the amplitude is equal to the stress from the external load. In the conditions of cyclic loading, the compressive residual stresses increase, and the tensile stresses decrease the fatigue strength. In this case, the residual stresses, regardless of their sign, relax in each loading cycle. The closer the value of the resultant stresses to the yield point, the faster the complete relaxation of the residual stress occurs. Proceeding from the above said, the tensor of the acting (total) stresses was represented as a sum of residual and fatigue stress tensors:

$$
\left[\mathrm{T} \sigma_{\mathrm{act}}\right]=\left[\mathrm{T} \sigma_{\mathrm{res}}\right]+\left[\mathrm{T} \sigma_{\mathrm{fat}}\right] \text {. }
$$

The first, second and third invariants were distinguished from the stress tensor, followed by the determination of the 
principal stresses, the intensity of the shearing stresses, and the index of the stress state scheme. In the framework of this paper, the index of the stress state scheme $\Pi$ was determined by the ratio of the mean normal stress $\sigma$ to the intensity of the shear stresses $\mathrm{T}$ in the form $\Pi=\sigma / \mathrm{T}$. In the subsequent calculations, it was assumed that the relaxation of the residual stresses and, accordingly, the change in the index of the scheme occur during the entire time of fatigue loading in accordance with the model obtained by statistical processing of the results of experimental studies:

$$
\sigma_{\text {res }}=\sigma_{\text {res } 0}+\frac{a}{\ln \left(b N^{c}\right)}
$$

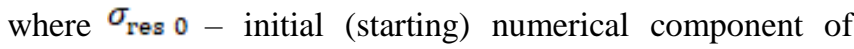
residual stresses in the chosen point; ${ }^{N}$ - current value of the number of cycles; ${ }^{a, b}, c$ - coefficients.

A model is developed for the accumulation of deformation and the depletion of metal plasticity reserves at the stage of cyclic fatigue life. According to this model, upon completion of machining, a residual (inherited) stress strain state takes place in the surface layer, characterized by the distribution of the degree of shear strain $\Lambda_{m}$ and the degree of depletion of the plasticity reserve $\Psi_{m}$ over the depth $h_{m}$, and also the residual stress tensor $\left[\mathrm{T} \sigma_{\mathrm{res}}\right]$, balanced within the part (Fig. 3 ). The load program was presented in known coordinates "П- $\Lambda$ " (Fig. 1). The start of the stage of cycle fatigue life means a cyclic change in the index of the stress state scheme at each point of the cross section of the part. Due to the non-uniform nature of the distribution, the component of the residual stress tensor stress scheme indices will vary from some maximal $\Pi+$ to some minimal
$\Pi^{-}$values in each section of the part under consideration. As the results of numerous studies show, there is a certain material point (particle) located in a certain section of the part and at a certain depth from the surface $h_{\text {crack }}$ where the appearance of traces of fracture (visible crack) is most likely. Apparently, this section will be the one in which the "hardest" loading scheme takes place. Therefore, it will be sufficient to estimate the accumulation of deformations and the depletion of the plasticity reserve in this section and precisely at this material point, called the point of probable destruction.

At a zero moment of time, fatigue stresses are absent. Then, at the start of the stage of cyclic fatigue life only residual stresses function, and the index of the stress state scheme is determined through the components of these (acting) stresses:

$$
\Pi_{\mathrm{D}}=\frac{\frac{1}{3} I_{1}\left[\mathrm{~T} \sigma_{\mathrm{res}}\right]}{\sqrt{\left|I_{2}\left[D \sigma_{\mathrm{res}}\right]\right|}}
$$

where $I_{1}\left[\mathrm{~T} \sigma_{\text {res }}\right]-$ the first invariant of the residual stresses tensor; $I_{2}\left[D \sigma_{\text {res }}\right]_{-}$the second invariant of residual stresses deviator.

Within the framework of the proposed model, the accumulation of deformations and the depletion of the plasticity of the surface layer metal occurs with the index of the stress state varying from cycle to cycle, and within each cycle. This is due, first of all, to relaxation of residual stresses and their complete disappearance at the time of formation of an apparent fatigue crack. At this point in time only fatigue stresses act in the dangerous section. The stage under consideration terminates with a complete relaxation of

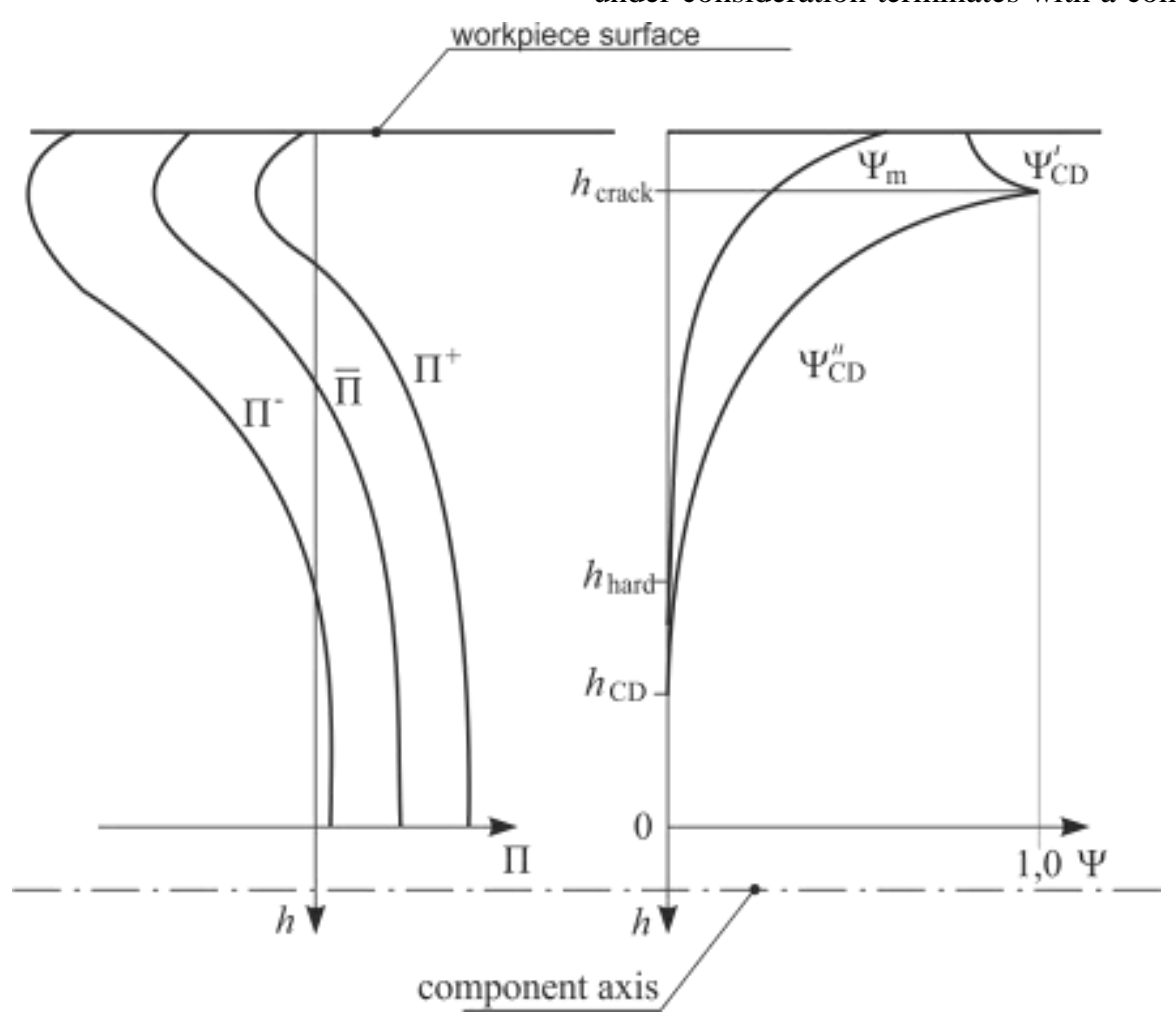

Fig. 1. Distribution of parameters of the stress-strain state along the depth of the hardened surface layer of the part in the process of cyclic loading 
the residual stresses and complete depletion of the plasticity reserve. At this point in time, at the point with the coordinate $h_{\text {crack }}$ the degree of plasticity reserve depletion is $\Psi_{\mathrm{CD}}=1$; there took place an increase in the degree of plasticity reserve depletion in the direction toward the surface of the part, which is described by the stress diagram $\Psi_{\mathrm{CD}}^{*}$; there was an increase in the degree of plasticity reserve depletion in the direction towards the axis of the part to the depth $h_{\mathrm{CD}}$, which is described by the stress scheme $\Psi_{\mathrm{CD}}^{\mathrm{n}}$. At this time, only the fatigue stresses function, and the value of the scheme index of the acting stress state is determined by the expressions:

$$
\Pi_{\mathrm{A}}=\frac{\frac{1}{3} I_{1}\left[\mathrm{~T} \sigma_{\mathrm{fat}}\right]}{\sqrt{\left|I_{2}\left[D \sigma_{\mathrm{fat}}\right]\right|}}= \pm 0,577,
$$

where $I_{1}\left[\mathrm{~T} \sigma_{\text {fat }}\right]-$ the first invariant of the fatigue stresses tensor; $I_{2}\left[D \sigma_{\text {fat }}\right]-$ the second invariant of fatigue stresses deviator. The values of the index of the stress state scheme obtained from the operating fatigue stresses at the finish of the stage of cyclic fatigue life correspond to the conditions of uniaxial tension and compression.

The surface layer of the part metal in its properties at this point in time is even more uneven. The boundary of this irregularity is the discontinuity in the form of a discontinuity (initial fatigue crack) of $0.02 \ldots 0.2 \mathrm{~mm}$ in size. The appearance of these visible defects means the beginning of a new stage - the stage of cyclic fracture strength.

\section{RESULTS AND DISCUSSION}

The fatigue tests were performed in 8 series of 8 samples in each series. The tests were carried out at a speed of rotation, the time of one revolution (cycle) is $t=1 / 50=0.02$ sec.; the amplitude of the stresses of the cycle was
$-280 \mathrm{MPa} \leq \sigma_{a} \leq+280 \mathrm{MPa}$.. The samples were made on a CNC machine, then the samples were annealed to remove the layer hardened by previous treatment. Next, they were machined on a CNC machine in the following mode: $\mathrm{n}=2000 \mathrm{rpm} ; \mathrm{S}=0.25 \mathrm{rpm} ; \mathrm{a}=1 \mathrm{~mm}$. The geometry of the cutting part of the tool made of the hard tungsten-cobalt alloy VK3 was as follows $\alpha=9^{\circ} ; \gamma=10^{\circ} ; \lambda=0 ; \varphi=45^{\circ} ; \varphi_{1}=30^{\circ}$; $\rho=0,03 \mathrm{~mm}$. The zero series of samples is the one after annealing and turning. Rolling of fatigue samples of 1 to 7 series with a working part diameter of $20 \mathrm{~mm}$ on a CNC machine was performed using the following modes: rolling force P-1000, 1500, 2000, 3000, 3500, 4000, $4500 \mathrm{~N}$ for the samples of 1 to 7 series, respectively; feed $\mathrm{S}=0.07 \mathrm{~mm} / \mathrm{rev}$; frequency $n=630 \mathrm{rpm}$; profile radius of the roller $=5 \mathrm{~mm}$.

In each series of samples one sample specimen was processed, on which the fixation of the zone of deformation was carried out. According to the parameters of the deformation zones, after cutting and surface-strain hardening, the finite element modelling of stress strain states was performed, and the calculation of the mechanical state of the metal of the surface layer along the streamlines was performed. At the same time, the calculated parameters of the mechanics after machining by cutting served as initial conditions for solving the hered itary task of Surface plastic strain hardening mechanics of SPS and fatigue loading. The point of probable destruction of the metal of the surface layer was determined experimentally and by calculation; at this point there is the most "rigid scheme" of the stress-strain state (the maximum level of tensile stresses at the maximum level of the degree of shear strain and the degree of depletion of the plasticity margin).

As an example, let us consider the laws of the mechanics of fatigue loading at the point of probable failure for the first series of tests (Fig. 2 and 3).

The dependence of the index of the stress state on the number of cycles is of a complex nature (Fig. 2). It is seen

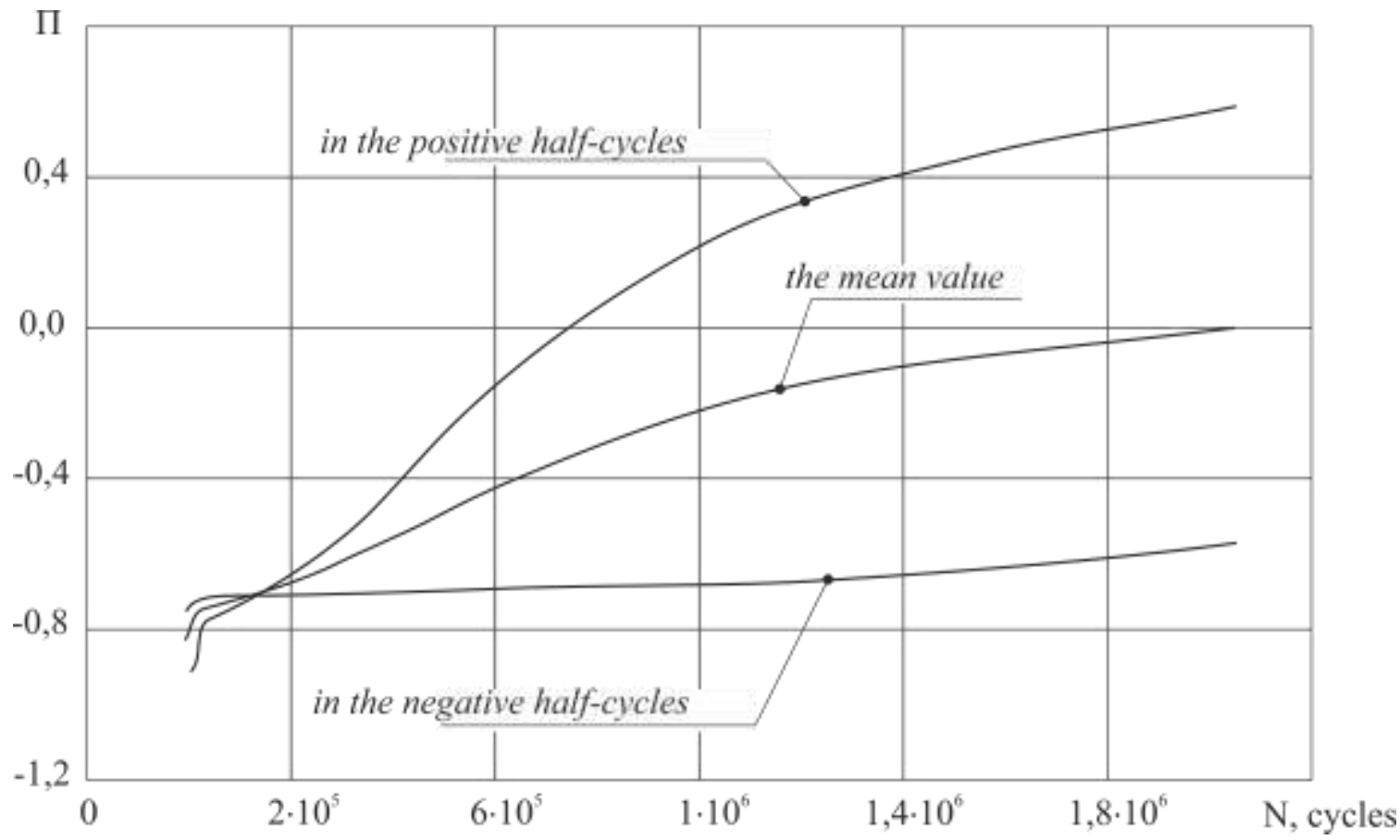

Fig. 2. Index of stress state scheme versus number of cycles 
that in the initial period the index of the scheme is negative, and its range relative to the mean value is relatively small. At $\mathrm{N} \approx 118500$ cycles, the range of the index scheme is close to zero. At $\mathrm{N}>118500$, there is a significant shift of the scheme index in the region of more "rigid" schemes in positive loading half-cycles, and insignificant - in the negative halfcycles of loading; at the same time, the range of this index is increasing relative to the mean value. The complete relaxation of residual stresses and the formation of an apparent crack occur at $-0.577 \leq \Pi \leq+0.577$, which corresponds to the schemes of simple uniaxial compression and tension, respectively.

Similar regularities have been found for all the test series studied. It can be argued that an increase in the number of cycles eventually leads to a more "rigid" scheme of the stress state and an increase in the range of the scheme index in each loading cycle. In addition, in the 5 th to 7 th series there is a more pronounced tendency in changing the index of the scheme at $\mathrm{N}>500,000$ cycles of loading. The analysis showed that the index of the scheme varies within wide limits during each of the selected cycles of fatigue loading. Despite the symmetrical cycle of loading stresses due to the influence of residual stresses, the change in the scheme index has an asymmetric character (Fig. 3).

The technological inheritance is expressed in the fact that

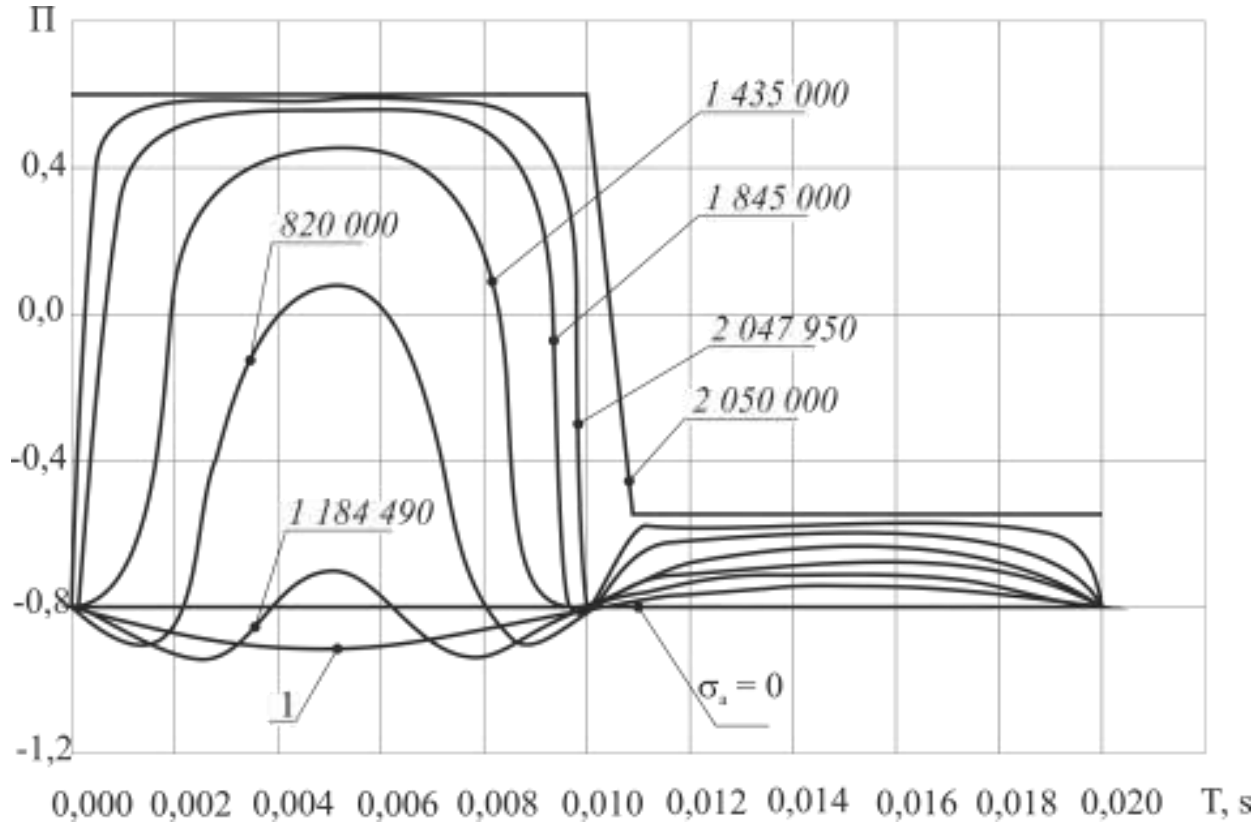

Fig. 3. Change of the index of stress state scheme within one cycle; the figures show the number of the fatigue loading cycle

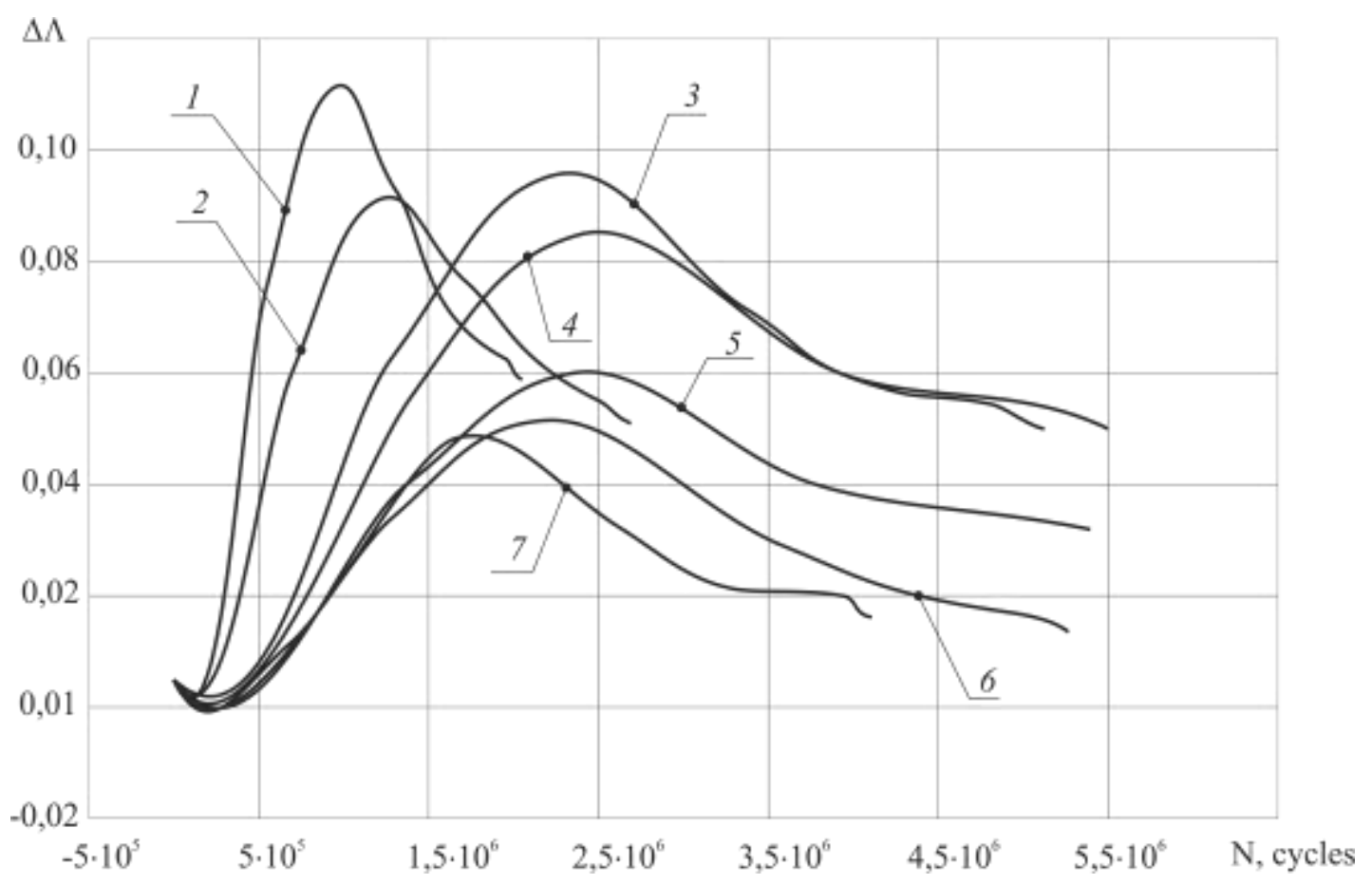

Fig. 4. Instantaneous shear deformation versus the number of cycles of fatigue loading: the figures indicate the numbers of series 
the level of instantaneous deformations varies with cycling (Fig. 4). It is seen that the greatest increment in strain for the 1 -st series occurs with the number of cycles $N=600000$ 1000000. An increase in the level of hereditary deformation (series 2-4) leads to a decrease in the absolute instantaneous values of deformation and their displacement to the region of cycles $N=1200000-2500000$. Further increase in the level of deformation accumulated during machining further reduces the level of absolute instantaneous deformation values and shifts them to the region of smaller loading cycles $N=2400000-1700000$ (5-7 series).

The loading history affects the rate of strain accumulation. With increasing hereditary deformation, the program area of fatigue loading narrows, which is expressed in a more shallow character, less deformation accumulated at the stage of cyclic fatigue life at a higher rate of change of the stress state index in each cycle and displacement of the entire loading program into the region of "harder" values. The absence of initial hardening leads to a "rigid" loading scheme with a shorter cyclic fatigue life of the product. In addition, an increase in the level of hereditary deformation leads to the fact that the fatigue loading program starts with more "rigid" schemes. This again speaks about the unified character of the impact of the loading history on the subsequent accumulation of deformations, regardless of the method of action on the surface layer of the product (cutting, surface plastic strain hardening, cyclic loading): the greater the inherited deformation, the more the loading program in the subsequent stage shifts to the region of "harder" values.

The loading history also determines the general nature of the accumulation of deformation at the stage of cyclic fatigue life (Fig. 5). The higher the hereditary deformation, the smaler the accumulated deformation at the stage of cyclic fatigue life and the smaller the increment of the degree of deformation in one loading cycle. The degree of shear deformation accumulated in the cycle is determined by the established loading program, and it is not constant, it varies depending on the history (including fatigue) of loading. Within each cycle the monotony of deformations accumulation and of the depletion of the plasticity reserve is broken 2 to 5 times.

Despite the fact that the loading history has an effect on the fatigue life and the rate of deformation accumulation in each cycle, nevertheless, the process of depletion of plasticity reserve obeys the regularities common with mechanical processing.

\section{CONCLUSION}

(1) The device of the mechanics of technological inheritance is used to describe the accumulation of deformations and the depletion of plasticity reserve at the stage of cyclic fatigue life from the level reached after machining. The index of the stress state scheme, unified in structure and physical meaning, is proposed for various stages and loading phases - machining by cutting and surface plastic strain and fatigue loading. The stress state index is calculated from the tensors of hereditary residual stresses and stresses and fatigue loading.

(2) It is shown that the surface layer of the article operates under conditions of cycle asymmetry which is the greater, the greater the residual stresses. In this case, the residual stresses, regardless of their sign, relax in each loading cycle.

(3) A model is developed for the accumulation of deformation and the depletion of the plasticity reserve

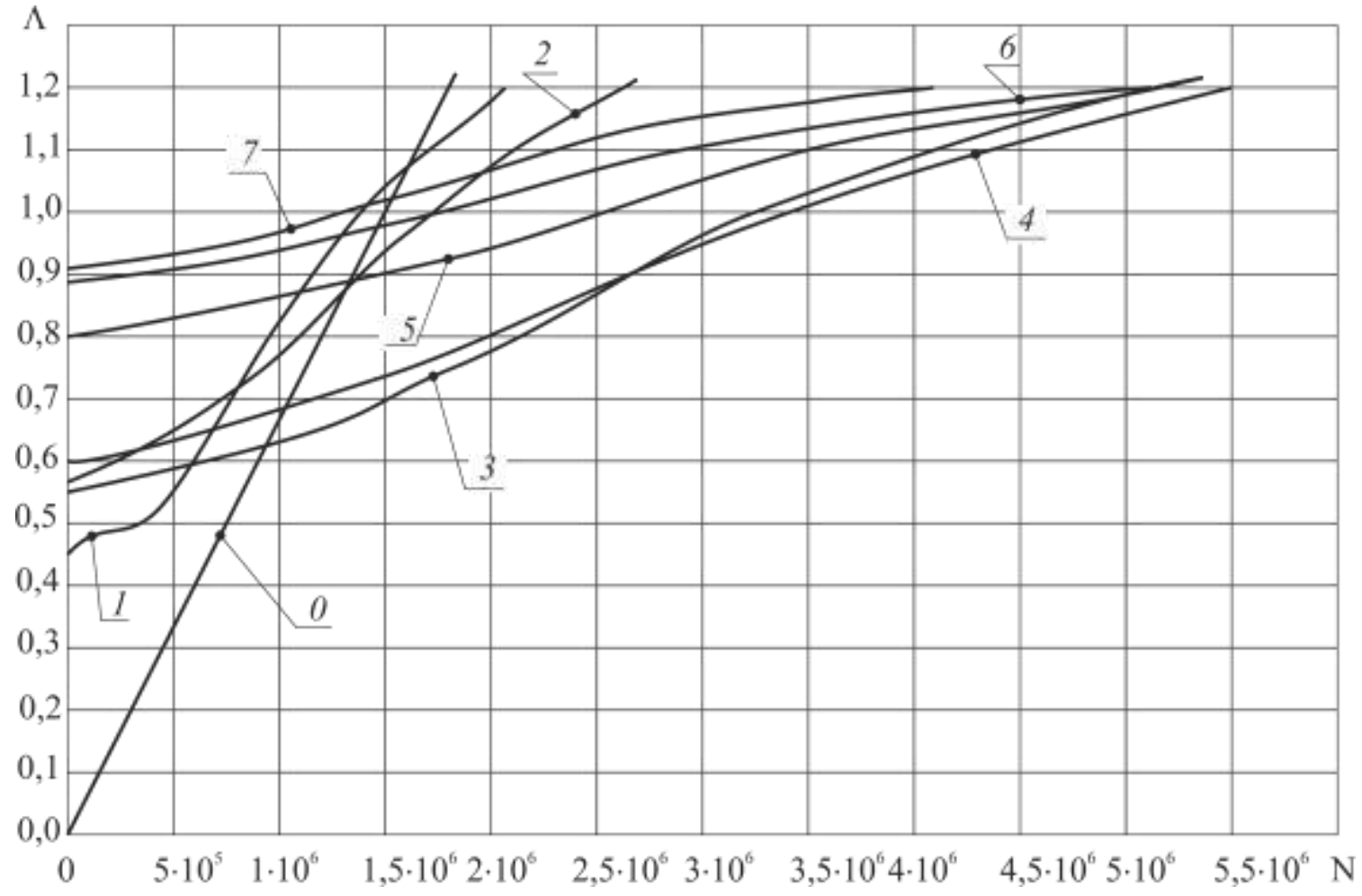

Fig. 5. The accumulated degree of shear deformation versus the number of cycles of fatigue loading: the figures indicate the numbers of series 
of the metal at the stage of cyclic fatigue life, within the framework of which the accumulation of deformations and the depletion of the plasticity reserve of the metal of the surface layer occurs with the index of stressed state scheme varying from cycle to cycle, and within each cycle.

(4) Experimental studies of the influence of technological inheritance on the state of the surface layer at the stage of fatigue loading are performed. The influence of loading history on the character of accumulation of small (inelastic) plastic deformations at the stage of cyclic fatigue life is established. A complex nonmonotonic character of the change in the stress state and the corresponding accumulation of deformations and the depletion of the plasticity reserve in each cycle of fatigue loading is shown.

(5) Calculations of deformation parameters at the stage of cyclic fatigue life based on the inherited degree of shear deformation and the degree of depletion of plasticity reserve are performed. It is shown that the developed calculation technique taking into account the loading history has shown a high reliability.

\section{REFERENCES}

[1] Zvonarev I.E., Ivanov S.L., Shishlyannikov D.I., Fokin A.S. "Studies of surface hardness of metal in the areas of increased wear and destruction of mining machine parts," Vestnik PNIPU. Geology. Oil and gas and mineral mining, 2014, 11: 67-76.

[2] Kogayev V.P. "Calculations for strength at stresses variable in time," Moscow. Mechanical Engineering, 1993.

[3] Makhutov N.A. "Strength and safety: fundamental and applied studies," Novosibirsk: Nauka, 2008.

[4] Karzov G.P., Margolin B.Z., Shvetsova V.A. "Physical and mechanical modelling of destruction processes," St. Petersburg: Polytekhnika, 1993.

[5] Mozharovskiy M.S., Bobyr' N.I. "Fatigue life and material plasticity at cyclic loading along various trajectories depending on the type of loading prehistory, " Problems of strength, 1979, 12: 1-5.

[6] Gusev A.S. "Fatigue resistance and durability of structures at sudden loads," Moscow. Mechanical Engineering, 1989.

[7] Romanov A.N. "Selection of material in designing. Kinetic criteria of destruction at cyclic loading at the stages of cracks formation and development,“ Surface engineering. Supplement to Journal "Spravochnik. Inzhenernyi zhournal", 2001. 5: 33-38.

[8] Guan D.A , Sun Q.A , Yang F.B. "A modified low cycle fatigue damage model for metals," Guti Lixue Xuebao/Acta Mechanica Solida Sinica, 2013, 34 (6): 571-578.
[9] Kourousis K.I. "A cyclic plasticity model for advanced light metal alloys," Applied Mechanics and Materials, 2013, 391: 3-8.

[10] Shanyavskiy A.A. "Mechanisms and modeling of subsurface fatigue cracking in metals," Engineering Fracture Mechanics, 2013, 110:350363.

[11] Mikheevskiy S.A , Glinka G.A , Lee E.B. "Fatigue crack growth analysis under spectrum loading in various environmental conditions," Metallurgical and Materials Transactions A: Physical Metallurgy and Materials Science, 2013, 44 (3): 1301-1310.

[12] Kogaev V.P., Makhutov N.A., Gusenkov A.P. "Calculations of machine and structure parts for strength and fatigue life: Spravochnik," Moscow. Mechanical Engineering, 1985.

[13] Troschenko V.T., Zhabko N.I. "Studies of influence of residual stresses on regularities of non-elastic cyclic deformation of metals in conditions of non-uniform stressed state," Problems of Problems of strength, 1979, 8: 25-29.

[14] Suslov A.G., Blumenstein V.Yu., Gurov R.V. and others. "Technology and tools for finishing-hardening of parts by surface plastic deformation," Moscow. Mechanical Engineering, 2014.

[15] Smelyanskiy V.M. "Mechanics of parts hardening by surface plastic strain," Moscow. Mechanical Engineering, 2002.

[16] Gryadunov I.M., Radchenko S.Yu., Dorokhov D.O. \& Morrev P.G. "Deep Hardening of Inner Cylindrical Surface by Periodic Deep Rolling - Burnishing Process,“ Modern Applied Science, 2015, 9(9): 251. doi:10.5539/mas.v9n9p251

[17] Majzoobi G.H. \& Jouneghani F. Zare \& Khademi E. "Experimental and numerical studies on the effect of deep rolling on bending fretting fatigue resistance of A17075,“ Int J Adv Manuf Technol. 2016, 82:2137-2148. doi:10.1007/s00170-015-7542-Z

[18] Denkena, B., Grove, T., Maiss, O. "Influence of hard turned roller bearings surface on surface integrity after deep rolling," 3rd CIRP Conference on Surface Integrity (CIRP CSI). doi: 10.1016/j.procir.2016.02.047

[19] Vielma A.T., Llaneza V. and Belzunce F.J. "Shot peening intensity optimization to increase the fatigue life of a quenched and tempered structural steel,“ Procedia Engineering, 2014, 74:273-278. doi: 10.1016/j.proeng.2014.06.261

[20] Abrão A.M., Denkena B., Köhler J., Breidenstein B. and Mörke T. "The influence of deep rolling on the surface integrity of AISI 1060 high carbon steel,“ 2nd CIRP Conference on Surface Integrity (CSI). doi: 10.1016/j.procir.2014.04.006

[21] Blumenstein V.Yu., Smelyanskiy V.M. "Mechanics of technological inheritance at the stages of machining and operation of parts of machines, “Moscow. Mechanical Engineering-1, 2007.

[22] Blumenstein V. "Mechanics of Technological Inheritance, "International journal for science and innovations for the industry Innovations in discrete productions issue, 2015, 2/2015(2): 18-21.

[23] Mahalov M.S., Blumenstein V.Yu. "Finite Element Surface Layer Inheritable Condition Residual Stresses Model in Surface Plastic Deformation Processes,“ IOP Conf. Series: Materials Science and Engineering, 2016, 126:012004. doi:10.1088/1757$899 \mathrm{X} / 126 / 1 / 012004$ 